\title{
Spatial Variations of Phytochemistry in Salvia Miltiorrhiza and Environmental Factors
}

\author{
Xiaoyu Chen ${ }^{1}$, Chao He ${ }^{1}$, Jie Cui ${ }^{1}$, Binbin Yan ${ }^{2}$, Yeye Geng ${ }^{3}$, Junling Hou ${ }^{3 *}$, Wenquan Wang ${ }^{1,3,4}$ and \\ Weixu Chen ${ }^{5}$ \\ ${ }^{1} 1$ Institute of Medicinal Plant Development, Chinese Academy of Medicinal Sciences and Peking Union Medical Collage, Beijing, China \\ ${ }^{2}$ College Pharmacy, Chengdu University of Traditional Chinese Medicine, Chengdu, China \\ ${ }^{3}$ School of Chinese Materia Medica, Beijing University of Chinese Medicine, Beijing, China \\ ${ }^{4}$ Engineering Research Center of Good Agricultural Practice for Chinese Crude Drugs, Ministry of Education, Beijing, China \\ ${ }^{5}$ Shang yao hua yu (LinYi) Tradtional chineese Medicine Resouces Co.,Ltd, Shandong, China \\ *Corresponding author: Wenquan Wang, Institute of Medicinal Plant Development, Chinese Academy of Medicinal Sciences and \\ Peking Union Medical Collage, Beijing, China \\ Junling Hou, School of Chinese Materia Medica, Beijing University of Chinese Medicine, Beijing, China
}

\begin{abstract}
ARTICLE INFO
Received: 幽 December 03, 2019

Published: 㓞January 08, 2020

Citation: Xiaoyu Chen, Chao He, Jie Cui, Binbin Yan, Yeye Geng, Junling Hou, Wenquan Wang, Weixu Chen. Spatial Variations of Phytochemistry in Salvia Miltiorrhiza and Environmental Factors. Biomed J Sci \& Tech Res 24(3)-2020. BJSTR. MS.ID.004051.

Abbreviations: SAB: Salvianolic Acid B; RA: Rosmarinic Acid; DTSI: Dihydrotanshinone I; CTS: Cryptotanshinone; TSI: Tanshi-

ABSTRACT

To provide a basis for controlling the quality of Salvia miltiorrhiza Bunge under artificial cultivation, the present work was designed to evaluate the fingerprinting and six major bioactive compounds, that is, salvianolic acid B (SAB), rosmarinic acid (RA), dihydrotanshinone I (DTSI), cryptotanshinone (CTS), tanshinone I (TSI) and tanshinone IIA (TSIIA), in major production areas in China and analyse the correlations with such factors as climate, geography and soil. The results showed that RA and SAB responded similarly to the environment factors, and RA contributed more to the phytochemical differentiation and displayed significant positive correlations with $\mathrm{Ca}$ and $\mathrm{Tp}$ and negative correlations with monthly precipitation. DTSI, CTS, TSI and TSIIA responded similarly to the environment factors, of which, DTSI and TSI were significantly correlated with environmental factors. DTSI and TSI displayed negative correlations with monthly and annual average temperature and DTSI displayed positive correlation with $\mathrm{Mn}$ and $\mathrm{Zn}$. It was concluded that chemical variations of S. miltiorrhiza were strongly correlated with soil, and phenolic acids displayed significant negative correlations with monthly precipitation and tanshinones displayed significant negative correlations with environmental changes of temperature.
\end{abstract} none I; TSIIA: Tanshinone IIA; PCA: Principal Component Analysis; AP: Available Phosphorus; AN: Available Nitrogen
Keywords: Bioactive Components; Climate; Geography; Soil Characteristics

\section{Introduction}

Salvia miltiorrhiza Bunge (Danshen in Chinese) is a traditional Chinese medicine which is used to treat cardiovascular disease, cerebrovascular disease, and other diseases [1,2], and its main two bioactive components, terpenoids and phenolic acids, contribute to these remarkable functions [3]. Phenolic acids (e.g., salvianolic acid $\mathrm{B}(\mathrm{SAB})$ and rosmarinic acid (RA)) have attracted research interest due to their antioxidant, antiatherosclerosis, and neuroprotective activities [4-6]. Tanshinones is one group of lipid-soluble bioactive ingredients in S. miltiorrhiza, which has a range of pharmacological effects, such as anticancer, antioxidant, antibacterial, and antiinflammatory activities [7-11]. The tanshinones mainly include dihydrotanshinone I (DTSI), cryptotanshinone (CTS), tanshinone I (TSI) and tanshinone IIA (TSIIA) [12]. Because of these efficacies and bioactivities, $S$. miltiorrhiza has been an important medicinal plant used both in Chinese medicinal formulas. Despite its long history of massive cultivation, much remains to be learned about the origin of phytochemical variation in $S$. miltiorrhiza, which is not only of theoretical significance in understanding the biological 
functions of metabolites and their evolution but also critical for quality control of $S$. miltiorrhiza in good agricultural practice.

It is known that medicinal plants have developed their own defense system by producing secondary metabolites which are important sources for pharmacological active products in response to environmental stresses $[13,14]$. A variety of environmental factors, such as climate, geographic distribution, radiation, and soil nutrition, have been proven to significantly influence the secondary metabolite profile [15-18]. There have been also some researches about influence of the ecological factors on the bioactive ingredients of $S$. miltiorrhiza $[19,20]$, but which mostly focus on single factor effect. For example, the study of WANG Wei et al. [21] on the effect of soil moisture on contents of $S$. miltiorrhiza which reported an appropriate drought stress during cultivation is beneficial for the raise of phenolic acids. The changes of key enzyme gene expression of rosmarinic acid biosynthesis pathway during heat stress was studied [22]. And the study of YU yange et al. [23] on the relationship between inorganic elements in wild-growing plants of $S$. miltiorrhiza and its soil. Additional investigations into the metabolic profile of plant populations of one species inhabiting varied environments are therefore necessary to understand the complex relationships between secondary metabolites and the habits in which they are produced [18].

It was proposed that global climate change influenced overall ecosystem functions, including the secondary metabolic profiles of plants. Our previous study also implied that the local environment may play an important role in the quality of cultivated S. miltiorrhiza [24]. This raised our interest to test whether the environmental parameters influence the phytochemical diversity in cultivated S. miltiorrhiza, and which is/are the most contributive one(s). Therefore, we enlarged our sampling to include five main regions of cultivation of $S$. miltiorrhiza in China with more plants, as well as the corresponding climatic and geographic data and soil samples, aiming to further investigate the secondary metabolic variation of cultivated $S$. miltiorrhiza among geographical regions and assess the effects of different environmental variables on phytochemical variation in terms of both the overall chromatographic fingerprint and six single major compounds of $S$. miltiorrhiza. This work will provide a reference for controlling the quality of $S$. miltiorrhiza during cultivation.

\section{Materials and Methods}

\section{Sampling}

Five major cultivation regions for $S$. miltiorrhiza throughout eastern and central China were included for sampling in November 2017. According to the sampling principle, three quadrats were set for each sampling sites, and five batches of plant samples were collected for each quadrat according to the diagonal cross. The identity of the plants was authenticated by Professor Wenquan Wang of Institute of Medicinal Plant Development. Soil samples are collected diagonally from each quadrangle. Geographic coordinates and altitude were recorded using a global positioning system see details in Table 1 \& Figure 1.

\section{Phytochemical Analyses with HPLC}

The plant samples were dried uniformly in drying ovens at $40^{\circ} \mathrm{C}$ and crushed after $0.355 \mathrm{~mm}$ mesh sieve. The phytochemical diversity of $S$. miltiorrhiza was presented in terms of variations of both overall chromatographic fingerprints and major single compounds. The present methods followed those in Gao et al. [25] with minor modification. Analyses were performed on an Thermo Scientific DionexUltiMate 3000 Series liquid chromatograph, consisting of Ulimate 3000 Pump and a Ulimate 3000 Autosampler connected to a Ulimate 3000 Variable UV detector. The reference material for RA, SAB, DTSI, CTS, TSI and TSIIA were obtained from Shanhai Winherb Medical Techonligy Co.,Ltd. All reference material had a purity of at least $98 \%$. An accurately weighed sample powder $(0.5 \mathrm{~g})$ was ultrasonically extracted with $50 \mathrm{~mL}$ of $75 \%$ methanol for $10 \mathrm{~min}$. The solutions were filtered through a $0.22 \mu \mathrm{m}$ membrane before HPLC analysis. The chromatographic separation was performed on an HPLC WondaSil C18 column $(4.6 \mathrm{~mm} \times 250 \mathrm{~mm}, 5 \mu \mathrm{m})$ using a gradient of methanol (A) and $0.01 \%$ aqueous phosphoric acid (B). The gradient program was performed as follows: 0-4 min, 35\% A; 4-12 min, 35-39\% A; 12-30 min, 39-45\% A; 30-35 min, 45-75\% A; 35-40 min, 75\% A; 40-65 min, 75-85\%, with flow rate of $1 \mathrm{~mL} \cdot \mathrm{min}-1$, column temperature of $30{ }^{\circ} \mathrm{C}$, and wavelength of $270 \mathrm{~nm}$. The six major compounds presented on the chromatograms of $S$. miltiorrhiza, including two hydrophilic Phenolic acids (RA and SAB) and four lipophilic diterpene (DTSI, CTS, TSI and TSIIA), were identified and quantified by corresponding reference compounds.

\section{Soil Analyses}

The collected soil samples were air-dried before screening through 40 mesh. The following attributes of each sample were analyzed: $\mathrm{pH}$, content of available nitrogen (An), available phosphorus (Ap) and available potassium (Ak). Soil analyses were performed according to the method of Bao [26]. Six microelements, $\mathrm{Fe}, \mathrm{Mn}, \mathrm{Ca}, \mathrm{Cu}, \mathrm{Zn}, \mathrm{B}$ and content of total nitrogen ( $\mathrm{Tn}$ ), total phosphorus ( $\mathrm{Tp}$ ) and total potassium ( $\mathrm{Tk}$ ) were provided by Chinese medicine resources geospatial grid information database at Beijing (http://www.tcm-resources.com/).

\section{Climatic Data}

Annual and monthly average temperature and precipitation were respectively acquired from the weather stations nearest the sampling sites, which are available from Chinese medicine resources geospatial grid information database at Beijing (http:// www.tcm-resources.com/).

\section{Statistical Analyses}

We examined the environmental correlations with both chromatographic fingerprints and the six single compounds based on comparisons within each set of the parameters. We extracted 15 characteristic peak areas comprising the fingerprint of each plant 
sample from the chromatograms. The variation of chromatographic fingerprints was computed via Cosine similarity comparison and principal component analysis (PCA) using SPSS19.0. Oneway ANOVA (multiple comparison) was performed to determine the statistical significance of content differences of the six major chemicals among the samples from different regions using SPSS 19.0. The test of homogeneity of variances was performed for the data before multiple comparisons, if equal variances are assumed, selected Duncan as post hoc test method; otherwise, selected TamhaneT2. Furthermore, redundancy analysis and PLS regression analysis were carried out using Canoco5.0 and Simca-P 12.0 software packages, respectively, to indicate correlation and contribution of the environmental factors with/to chromatographic fingerprints of $S$. miltiorrhiza. Pearson correlation analysis was applied to test the correlations between the six major components and environmental parameters using SPSS 19.0. The soil characters and environmental differentiation were also analyzed via ANOVA and PCA, respectively.

\section{Results}

\section{Climatic, Geographic, and Soil Characteristics of the} Cultivation Regions

S. miltiorrhiza has strong growth suitability and is widely distributed. The regions where the cultivated samples were

Table 1: Detailed Information of All Sampling Sites.

\begin{tabular}{|l|l}
\hline Sample-2orion & \\
\hline
\end{tabular}

\begin{tabular}{|c|c|c|c|c|}
\hline Sample & Cultivation Region & Altitude/m & Longitude $/^{\circ}$ & Latitude $/^{\circ}$ \\
\hline HB1 & Lingshou County, Hebei Province & 427.2 & 114.0869 & 38.4852 \\
\hline HB2 & Anguo City, Hebei Province & 64.4 & 115.2562 & 38.3563 \\
\hline SD1 & Laiwu City, Shandong Province & 288.6 & 117.7865 & 36.3199 \\
\hline SD2 & Laiwu City, Shandong Province & 353.1 & 117.8241 & 36.3167 \\
\hline SD3 & Kongcun Town, Shandong Province & 82.3 & 116.4586 & 36.1574 \\
\hline SD4 & Xiaozhi Town, Shandong Province & 136.1 & 116.4112 & 36.0925 \\
\hline SD5 & Zhucheng City, Shandong Province & 112.7 & 119.0296 & 36.0133 \\
\hline SD6 & Kushan Town, Shandong Province & 127.9 & 118.9676 & 35.9172 \\
\hline SD7 & Chengnan Town, Shandong Province & 134.4 & 118.8013 & 35.5394 \\
\hline SD8 & Xintai City, Shandong Province & 192.5 & 117.7715 & 35.8211 \\
\hline SD9 & Mengyin County, Shandong Province & 132.6 & 117.8537 & 35.6971 \\
\hline SD10 & Fangcheng Town, Shandong Province & 135.2 & 117.5583 & 35.6752 \\
\hline SD11 & Fangcheng Town, Shandong Province & 209.8 & 117.5606 & 35.6714 \\
\hline SD12 & Baotai Town, Shandong Province & 210.3 & 117.7545 & 35.5813 \\
\hline SD13 & Bolin Town, Shandong Province & 206.6 & 117.7446 & 35.5525 \\
\hline SD14 & Wenshui Town, Shandong Province & 84.2 & 117.7149 & 35.4166 \\
\hline SX1 & Yaozhou District, Shanxi Province & 783.8 & 108.9661 & 34.9479 \\
\hline SX2 & Yaozhou District, Shanxi Province & 903.4 & 108.8015 & 34.8921 \\
\hline SX3 & Yaozhou District, Shanxi Province & 892.4 & 108.808 & 34.8808 \\
\hline SX4 & Tongguan County, Shanxi Province & 345.8 & 110.1827 & 34.6206 \\
\hline SX5 & Huayin City, Shanxi Province & 352.6 & 110.135 & 34.5947 \\
\hline SX6 & Luonan County, Shanxi Province & 531.7 & 110.1426 & 34.0921 \\
\hline SX7 & Shangzhou District, Shanxi Province & 724.9 & 109.9614 & 33.8432 \\
\hline
\end{tabular}

collected are located from $119.0296^{\circ} \mathrm{E}$ to $104.536373^{\circ} \mathrm{E}, 38.4852^{\circ}$ $\mathrm{N}$ to $31.0019^{\circ} \mathrm{N}$. The annual rainfall in these regions varied from 423 to $1141 \mathrm{~mm}$, and the average yearly temperature ranged from 11.7 to $17.0{ }^{\circ} \mathrm{C}$. Obvious distinctions in geographic factors exist between the sites. Sichuan (SC) is at the highest elevation in altitude and is at the minimum longitude, whereas Shandong (SD) is at the lowest altitude and the maximum longitude. In latitude, Hebei (HB) is at the maximum latitude, whereas SC is at the minimum (Table 1 \& Figure 1). These distinctions result in climatic differences among regions. The sampling sites in SC had the highest annual average temperature and precipitation, whereas those in HB were the opposite. The monthly average temperatures were highest in SC, with January to April and October to December had a significantly higher level, except June, July and August were highest in Henan (HN). The soil was slightly alkaline in most sites, except for HB. None of the regions were deficient in nutrients, and the soil in HB and SC exhibited distinctive high concentration of available nitrogen, and the concentration of available potassium in HB was significantly higher. The concentration of organic matter in $\mathrm{HN}$ and SC was significantly lower. PCA of climatic and soil factors showed that the environment of the Shanxi (SX) site was closest to the HN site, these sites were distributed in the center of the plot, whereas the HB site was near to the SD site and the SC site was the most distal site (Figure 2). The determinant bringing about these results was temperature, and the next was precipitation. 


\begin{tabular}{|c|c|c|c|c|}
\hline SX8 & Shangzhou District, Shanxi Province & 576 & 109.9013 & 33.8231 \\
\hline SX9 & Xiaoyi Town, Shanxi Province & 641.9 & 110.1535 & 33.7515 \\
\hline SX10 & Danfeng County, Shanxi Province & 621.8 & 110.3217 & 33.6973 \\
\hline SX11 & Eryuhe Town, Shanxi Province & 796.5 & 109.7117 & 33.5998 \\
\hline SX12 & Xiaohekou Town, Shanxi Province & 773.5 & 109.6592 & 33.5849 \\
\hline HN1 & Lingbao City, He'nan Province & 189.3 & 110.7378 & 34.5788 \\
\hline HN2 & Yichuan City, He'nan Province & 228.4 & 112.3238 & 34.3801 \\
\hline HN3 & Qinghe Town, He'nan Province & 302.2 & 112.9241 & 33.3621 \\
\hline HN4 & Yangji Town, He'nan Province & 193.5 & 112.9669 & 33.3174 \\
\hline HN5 & Xiaohoushan Town, He'nan Province & 109.7 & 113.1533 & 33.2398 \\
\hline HN6 & Xiaohoushan Town, He'nan Province & 109.6 & 113.1533 & 33.2398 \\
\hline HN7 & Wolong District, He'nan Province & 206.2 & 112.5016 & 33.1015 \\
\hline SC1 & Bazhong City, He’nan Province & 712.6 & 106.7607 & 31.8626 \\
\hline SC2 & Jiange County, Sichuan Province & 539.3 & 105.7533 & 31.6471 \\
\hline SC3 & Zitong County, Sichuan Province & 546.9 & 105.5779 & 31.3909 \\
\hline SC4 & Zhongjiang County, Sichuan Province & 637.5 & 104.574 & 31.1773 \\
\hline SC5 & Zhongjiang County, Sichuan Province & 899.3 & 104.5364 & 31.002 \\
\hline
\end{tabular}

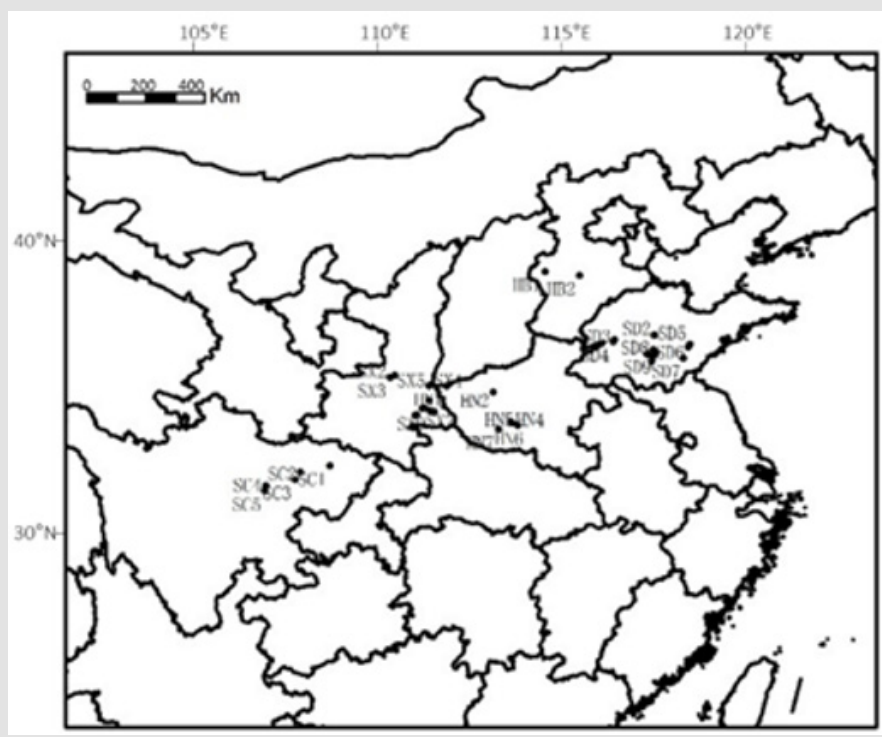

Figure 1: Sample location of S. miltiorrhiza.

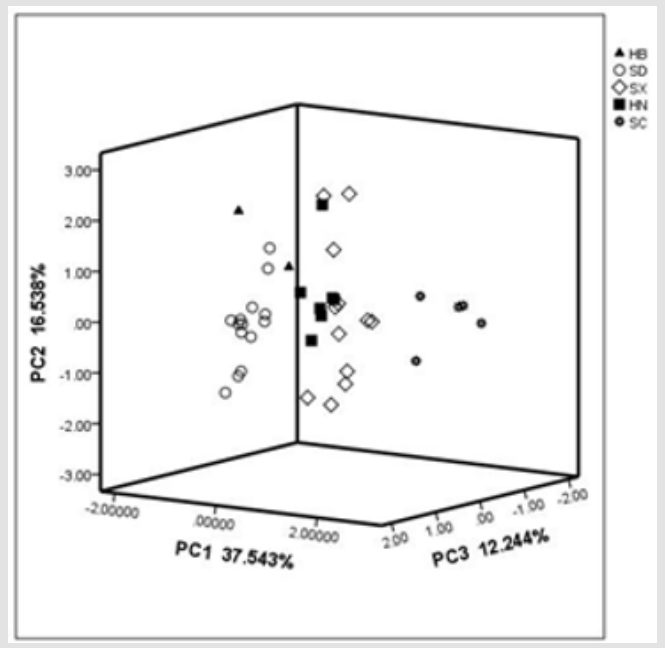

Figure 2: PCA plot for climatic and soil factors of 40 samples of S. miltiorrhiza from the five studied regions. 


\section{Phytochemical Variation of $S$. miltiorrhiza}

Most samples showed general consistency with the main model fingerprint (M40) in the Cosine similarity analysis (>0.90) (Table 2). However, the highest mean similarities of 0.959 and 0.947 were presented in the $\mathrm{HN}$ and SC population, respectively, with lesser deviations of 0.068 and 0.052 , indicating stable chemical profiles in these populations, whereas the SX population exhibited the lowest similarity of 0.878 with great deviation of 0.146 . Moderate values were generated in the HB and SD populations $(0.921 \pm 0.057$ and
$0.916 \pm 0.110$, respectively). We used hierarchical clustering method to analysis 40 samples of 15 common peak areas. Furthermore, the PCA demonstrated the contributing rate of three principal components reached $49.43 \%, 14.98 \%$ and $9.14 \%$, respectively and the PCA plot shows the SX site was closest to the SC site (Figure $3)$. The loading values of PCA indicated that the fifth, fourth and fifteenth (tanshinone IIA) peaks were the most contributive variable on the first axis and the second peak (RA) was the most on the second axis.

Table 2: Comparisons of Cosine Similarity between Each Plant Sample and the main model fingerprint of S. miltiorrhiza.

\begin{tabular}{|c|c|c|c|c|c|c|c|c|c|}
\hline Sample & $\begin{array}{c}\text { Similarity to } \\
\text { M40 }\end{array}$ & Sample & $\begin{array}{c}\text { Similarity to } \\
\text { M40 }\end{array}$ & Sample & $\begin{array}{c}\text { Similarity to } \\
\text { M40 }\end{array}$ & Sample & Similarity to M40 & Sample & $\begin{array}{l}\text { Similarity } \\
\text { to M40 }\end{array}$ \\
\hline HB1 & 0.88 & SD1 & 0.979 & SX1 & 0.644 & HN1 & 0.991 & SC1 & 0.962 \\
\hline \multirow[t]{13}{*}{ HB2 } & 0.961 & SD2 & 0.724 & $\mathrm{SX} 2$ & 0.649 & HN2 & 0.807 & SC2 & 0.995 \\
\hline & & SD3 & 0.828 & SX3 & 0.851 & HN3 & 0.965 & SC3 & 0.913 \\
\hline & & SD4 & 0.985 & SX4 & 0.986 & HN4 & 0.987 & SC4 & 0.874 \\
\hline & & SD5 & 0.837 & SX5 & 0.986 & HN5 & 0.989 & SC5 & 0.99 \\
\hline & & SD6 & 0.991 & SX6 & 0.881 & HN6 & 0.988 & & \\
\hline & & SD7 & 0.991 & SX7 & 0.985 & HN7 & 0.987 & & \\
\hline & & SD8 & 0.994 & SX8 & 0.657 & & & & \\
\hline & & SD9 & 0.996 & SX9 & 0.923 & & & & \\
\hline & & SD10 & 0.697 & SX10 & 0.987 & & & & \\
\hline & & SD11 & 0.821 & SX11 & 0.996 & & & & \\
\hline & & SD12 & 0.992 & SX12 & 0.996 & & & & \\
\hline & & SD13 & 0.993 & & & & & & \\
\hline & & SD14 & 0.995 & & & & & & \\
\hline mean $\pm S D$ & $0.921 \pm 0.057$ & mean $\pm S D$ & $0.916 \pm 0.110$ & mean $\pm S D$ & $0.878 \pm 0.146$ & mean $\pm S D$ & $0.959 \pm 0.068$ & mean $\pm S D$ & $0.947 \pm 0.052$ \\
\hline
\end{tabular}

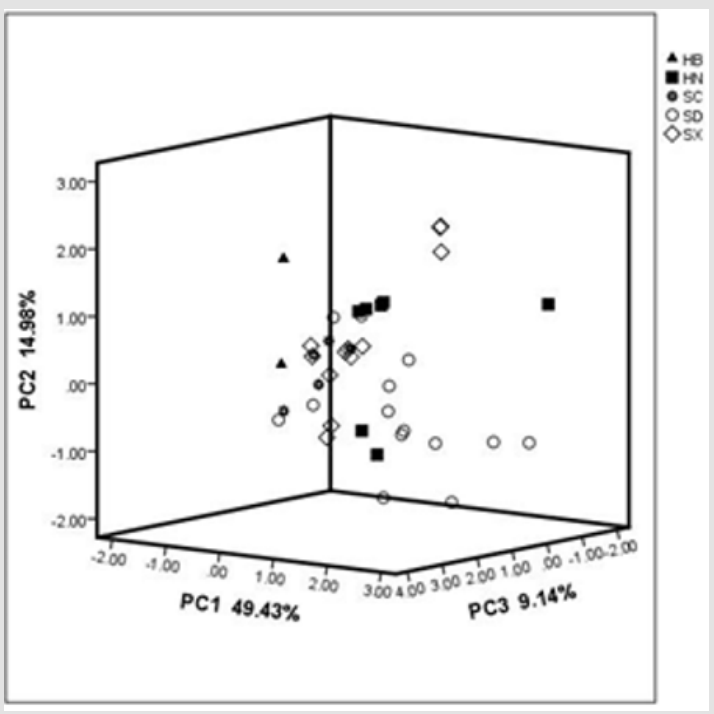

Figure 3: PCA plot for chromatographic fingerprints of 40 samples of S. miltiorrhiza from the five studied regions.

We further quantified the six main bioactive components, that is, rosmarinic acid (RA), salvianolic acid B (SAB), dihydrotanshinone I (DTSI), cryptotanshinone (CTS), tanshinone I (TSI) and tanshinone IIA (TSIIA) (Figures 4A-4F). RA, DTSI, CTS and TSI were present in significantly different amounts among regions as determined by one-way ANOVA $(\mathrm{p} \leq 0.05)$. Both the average content of DTSI and CTS were highest in the HB and HN population (DTSI 0.0613 and $0.0464 \%$, CTS 0.3280 and $0.2396 \%$, respectively) and lowest in 
the SC and SX population (DTSI 0.0067 and $0.0249 \%$, CTS 0.0984 and $0.0972 \%$, respectively). The SX population also showed the highest average content of RA (0.3410\%). The SC population showed the lowest average content of TSI (0.0492\%). Not significant regional variations in SAB and TSIIA were detected. In the SC and SX population, hydrophilic components were low, while lipophilic components were high, which were opposite in the SD and HN population.
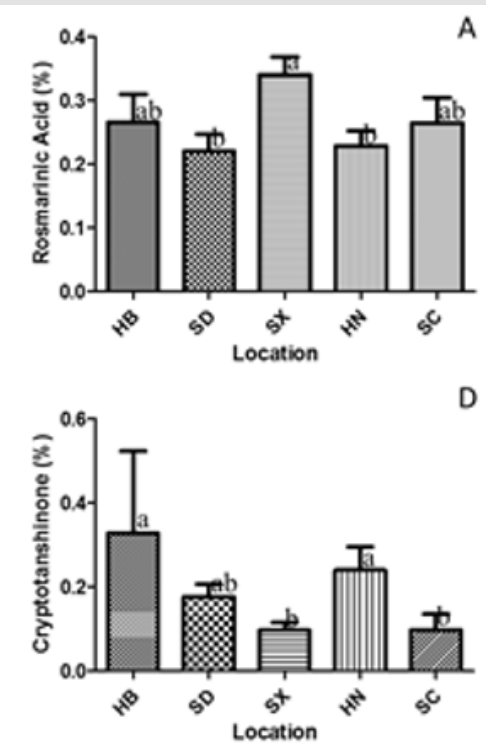

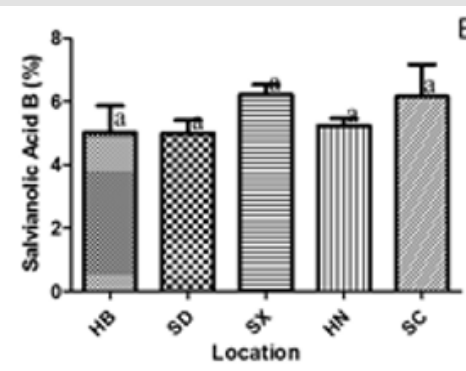

E

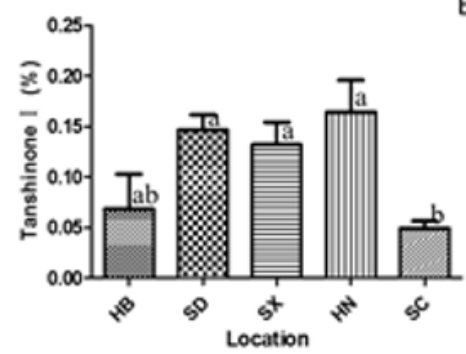

C
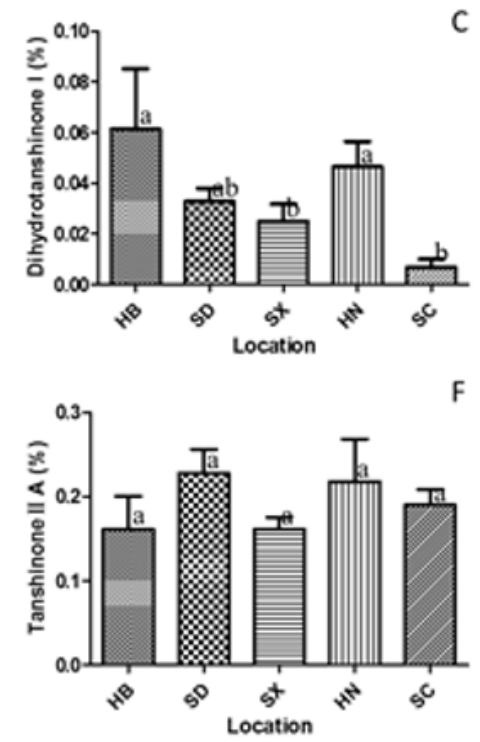

Figure 4: Content comparisons of rosmarinic acid

A. Salvianolic acid B

B. Dihydrotanshinone I,

C. Cryptotanshinone,

D. Tanshinone I

E. Tanshinone IIA

F. Among S. miltiorrhiza from different regions. (Error bars indicate standard deviations. Lowercase letters indicate significance at the level of 95\% resulted from post hoc multiple comparison of either LSD or Tamhane T2.).

\section{Correlations between Chemical Variations of S. miltiorrhiza and Environmental Variations}
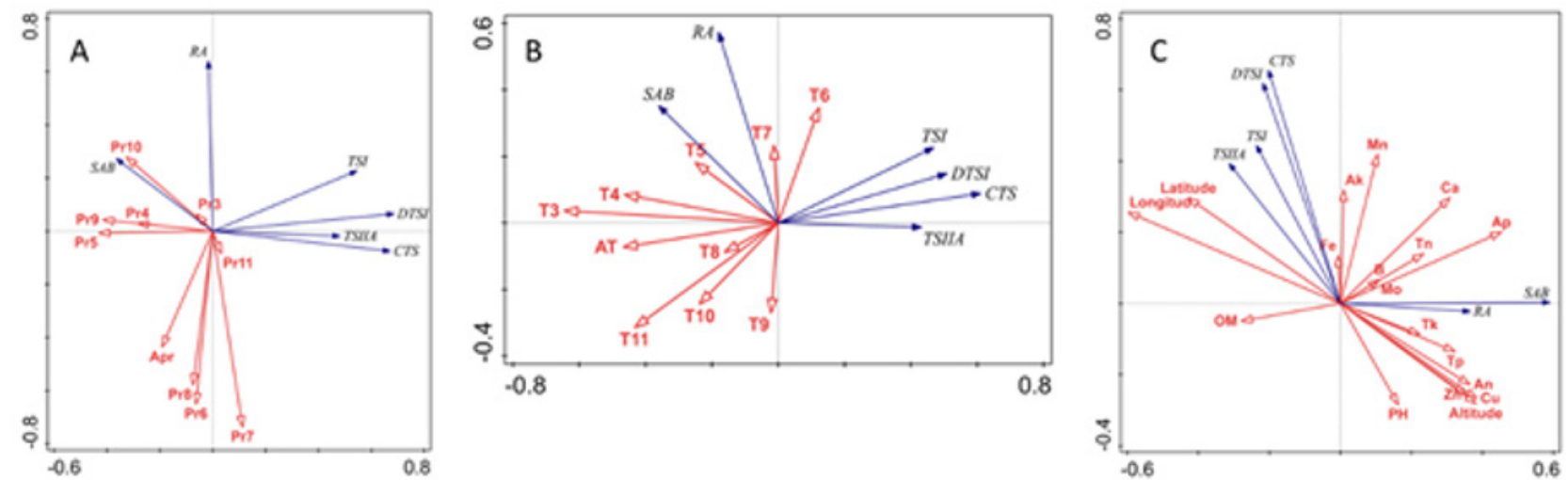

Figure 5: RDA plot showing the relationships between the contents of the six compounds of S. miltiorrhiza and precipitation

A. Temperature,

B. Soil factors

C. Pr, average monthly precipitation of each month; Apr, annual average precipitation; $\mathrm{T}$, average monthly temperature of each month; AT, annual average temperature; the subscript Arabic numbers stand for month; OM, content of organic matter; An, available nitrogen; Ap, available Phosphorus; Ak, available potassium; Tn, total nitrogen; Tp, total Phosphorus; Tk, total potassium. 
40 samples were applied to explore the correlations between environment and chemical diversity. The three plots of redundancy analysis (RDA) showed the relationships between the contents of the six compounds of $S$. miltiorrhiza and precipitation (Figure $5 \mathrm{~A}$ ), temperature (Figure 5B), soil factors (Figure 5C). In the Figure $5 \mathrm{~A}$, the correlation of ordination axis was more significant with the precipitation in July (Pr7), May (Pr5), October (Pr10) and September (Pr9). Pr7, June (Pr6) and August (Pr8) were negatively correlated with the hydrophilic compositions, especially RA. Pr5 and Pr9 were negatively correlated with the lipophilic compositions. In the Figure 5B, the average temperature in March (T3), April (T4) and annual average temperature (AT) were more closely related with the bioactive components of $S$. miltiorrhiza, which were negatively correlated with the lipophilic compositions. In the Figure 5C, geographic factors were more closely related with the bioactive components of $S$. miltiorrhiza. Longitude was negatively correlated with the hydrophilic compositions, which was positively correlated with lipophilic compositions. Latitude was also positively correlated with lipophilic compositions. Rather altitude was positively correlated with hydrophilic compositions, while negatively correlated with lipophilic compositions. Soil factors such as available phosphorus (Ap), available nitrogen
(An) and microelements include $\mathrm{Cu}$ and $\mathrm{Zn}$ were more closely related with bioactive components. $\mathrm{Cu}, \mathrm{An}$ and $\mathrm{Zn}$ were negatively correlated with lipophilic compositions.

In addition, on the basis of the contents of the six single compounds, Pearson correlation analysis revealed only RA and DTSI were significantly correlated with precipitation $(\mathrm{P}<0.05)$ and only lipophilic compositions such as DTSI and TSI were significantly correlated with average temperature $(\mathrm{P}<0.05)$, so only RA, DTSI and TSI were significantly correlated with a number of the environmental factors. To disclose the determining factors, the environmental variables with significant correlation above 0.3 were subject to analysis of partial least-squares (PLS) regression, which is a powerful tool for searching for key factors with an algorithm eliminating distinct collinearity among numerous variables that may result in poor stability of the model. There presents the standardized PLS regression coefficients with three components, RA, DTSI and TSI (Table 3). Precipitations in July, June (Pr7, Pr6) and Ca were computed to be the successive key factors affecting the content of RA. With regard to TSI, the most important variable was available nitrogen (An). The average temperature in March (T3), available potassium (Ak) and Mn were the influential variable to DTSI.

Table 3: Standardized PLS regression coefficients with three components, RA, DTSI and TSI.

\begin{tabular}{|c|c|c|c|c|c|c|c|c|c|c|}
\hline Factors & \multicolumn{2}{|c|}{ RA } & Factors & \multicolumn{2}{c|}{ TSI } & \multicolumn{2}{c|}{ DTSI } & \multicolumn{2}{c|}{ Factors } & \multicolumn{2}{c|}{ DTSI } \\
\hline & $\boldsymbol{\beta}$ & $\mathbf{p}$-value & & $\boldsymbol{\beta}$ & $\mathbf{p}$-value & $\boldsymbol{\beta}$ & p-value & \multicolumn{2}{|c|}{$\boldsymbol{\beta}$} & p-value \\
\hline Pr6 & -0.141 & $<0.01$ & $\mathrm{~T} 3$ & -0.105 & $<0.01$ & -0.075 & $<0.01$ & $\operatorname{Pr} 5$ & 0.074 & $<0.05$ \\
\hline Pr7 & -0.163 & $<0.01$ & $\mathrm{~T} 4$ & -0.099 & $<0.05$ & -0.056 & $<0.05$ & $\operatorname{Pr} 9$ & 0.073 & $<0.05$ \\
\hline Pr8 & -0.122 & $<0.05$ & $\mathrm{~T} 11$ & -0.094 & $<0.05$ & -0.062 & $<0.05$ & $\mathrm{Ak}$ & -0.056 & $<0.01$ \\
\hline Tp & 0.136 & $<0.05$ & AT & -0.094 & $<0.05$ & -0.059 & $<0.05$ & Mn & 0.059 & $<0.01$ \\
\hline Ca & 0.115 & $<0.01$ & An & -0.12 & $<0.01$ & & & Zn & 0.067 & $<0.05$ \\
\hline longitude & -0.114 & $<0.05$ & & & & & & longitude & 0.074 & $<0.05$ \\
\hline altitude & 0.118 & $<0.05$ & & & & & & latitude & 0.073 & $<0.05$ \\
\hline R2 & 0.37 & & R2 & 0.21 & & & & R2 & 0.26 & \\
\hline
\end{tabular}

\section{Discussion}

Our present results of PCA of fingerprint (Figure 3) and ANOVA of the contents of the single compounds consistently support the similar pattern of chemical differentiation of S. miltiorrhiza among the five regions. Chemical variation of S. miltiorrhiza in the SX and SC populations resembled each other, which were completely opposite to SD and HN populations, and HB population differed from others. The six single compounds analyzed responded differently to the environmental factors (Figure 5). Hydrophilic components, RA and SAB responded similarly to the environment factors, and RA was significantly correlated with more environmental factors. Lipophilic components, DTSI, CTS, TSI and TSIIA responded similarly to the environment factors, of which, DTSI and TSI were significantly correlated with most environmental factors. It has been suggested that $\mathrm{SAB}$ is derived from the RA pathway. Oxidative dimerization of hydroxystilbene occurs, and laccase has been proposed to catalyze the oxidative reaction from RA to SAB [27-29]. Tanshinones are synthesized via both the cytoplasmic mevalonate (MVA) pathway and plastidial methylerythritol phosphate (MEP) pathway [29-31].

The RDA, correlation and PLS analyses suggested that environmental variables, especially, soil factors, produced a significantly effect on the secondary metabolites in S. miltiorrhiza. Our results indicated negative correlation between lipophilic tanshinones and nitrogen, $\mathrm{Zn}$ and $\mathrm{Cu}$. And positive correlation between lipophilic tanshinones and potassium, Mn was detected. Ca produced positive correlation with RA. It is known that $\mathrm{N}, \mathrm{K}$ and $\mathrm{P}$ are important macronutrients for plants. $\mathrm{N}$ is the main constituent of proteins, chlorophyll, and enzymes involved in photosynthesis and $\mathrm{K}$ is needed for vital functions in metabolism, growth, and stress adaptation [32,33]. A S. miltiorrhiza plot experiment 
showed that plant growth and all the contents of the bioactive components responded negatively to increasing $\mathrm{N}$ availability, suggesting that $S$. miltiorrhiza is not a nitrophile [34]. And another plot experiment showed that the effects of the applications of $\mathrm{P}$ and $\mathrm{K}$ fertilizers at different growth stages on the root growth and bioactive compounds were shown to vary greatly in S. miltiorrhiza and $\mathrm{K}$ negatively affected the accumulations of tanshinones [35]. Microelements are very important to plants, so some scholars do studies about effect of microelements on S. miltiorrhiza. Wang B through sand culture experiments got that the mechanism of $\mathrm{Cu}$ and $\mathrm{Zn}$ on the accumulation of tanshinones may be that $\mathrm{Cu}$ and $\mathrm{Zn}$ improve the activity of peroxidase and polyphenol oxidase, which promote transformation of phenolic compounds to terpenes and therefore to increase contents of danshinones [36,37].

Mn increased the activities of PPO and POD, which promote the accumulation of tanshinones in S. miltiorrhiza [38]. So, if lack of these nutrition elements in the soil, we need to fertilizer these elements to guarantee the quality of $S$. miltiorrhiza. Precipitation also pronouncedly influenced hydrophilic components. Precipitation parameters displayed negative correlation with chemical variations of $S$. miltiorrhiza, particularly RA (Figure 5 \& Table 3). A plot experiment showed that the effects of drought stress on physiological characteristics were inhibitory, and drought stress could promote the accumulation of phenolic compounds [39]. A drought stress experiment showed drought stress is more advantageous to accumulate phenolic acid composition of $S$. miltiorrhiza lamina [40]. Water deficit has been reported to increase the expression of different genes involved in the biosynthesis of phenolic compounds [41-43]. Temperature also showed pronounced negative correlation with lipophilic components in S. miltiorrhiza, especially DTSI and TSI. Harpagoside displayed significant positive correlations with monthly and annual average temperature, which was related to heat tolerance [44]. High temperature and strong sunshine limited the content of ginsenosides through collecting Panax ginseng samples [45]. Despite reports on the effect of temperature factors on plant secondary metabolism in natural environments, an array of similar work has been carried out in controlled conditions.

In tomato plants, Anthocyanins showed pronounced increased levels when lowering the growth temperature from $24{ }^{\circ} \mathrm{C}$ to 18 ${ }^{\circ} \mathrm{C}$ or $12{ }^{\circ} \mathrm{C}$ [46]. Accordingly, the chemical variation of lipophilic components in S. miltiorrhiza might be related to cold tolerance. The pattern of differentiation of lipophilic components also corresponds to the longitudinal and latitudinal directions, which were negative with hydrophilic components. A positive correlation between latitude and chemical diversity was detected in juniper (Juniperus communis) needles [47]. Concentrations of anthocyanidin and delphinidin in Vaccinium myrtillus fruits varied significantly across latitude, with higher values from northern latitudes, whereas another anthocyanidin, cyanidin, was opposite [48]. Therefore, different plants or even different metabolites might differ in their responses to latitudinal variation [49]. Our result indicatied that the SC and SX populations were located at the lower latitude and longitude, and the other three were at higher longitude (Table 1). The factor of latitude comprises a series of other environmental factors, among which temperature and precipitation tend to fall with the growth of latitude.

\section{Conclusion}

Our results revealed how chemical differentiation in S. miltiorrhiza varied spatially and the regulating roles of environmental factors, but genetic and cultivational measures have not been considered in our study, which may also affect the metabolite composition of $S$. miltiorrhiza. However, further exploration of the insights of the present work by examination of the biological and biochemical relationships between the chemical differentiation and environmental factors, germplasms, and cultivational measures would be most interesting.

\section{References}

1. (2015) Chinese Pharmacopoeia Commission, Pharmacopoeia of the people's republic of chia (Volume I ). China Medical Science Press, Beijing, China, pp. 76-77.

2. Fei Y, Wang S, Yang L, Qiu Y, Li Y, et al. (2017) Salvia miltiorrhiza Bunge (Danshen) extract attenuates permanent cerebral ischemia through inhibiting platelet activation in rats. Journal of Ethnopharmacology 207: 57-66.

3. Kim R, Lee S, Lee CY, Yun H, Lee H, et al. (2018) Salvia miltiorrhiza enhances the survival of mesenchymal stem cells under ischemic conditions. Journal of Pharmacy and Pharmacology 70(9): 1228-1241.

4. Pang H, Wu L, Tang Y, Zhou G, Qu C, et al. (2016) Chemical Analysis of the Herbal Medicine Salviae miltiorrhizae Radix et Rhizoma (Danshen). Molecules 21(1): 51-62.

5. Zhou Y, Li W, Xu L, Chen L (2011) In Salvia miltiorrhiza, phenolic acids possess protective properties against amyloid $\beta$-induced cytotoxicity, and tanshinones act as acetylcholinesterase inhibitors. Environmental Toxicology and Pharmacology 31(3): 443-452.

6. Wing Shing Cheung D, Koon C, Ng C, Leung P, Fung K, et al. (2012) The roots of Salvia miltiorrhiza (Danshen) and Pueraria lobata (Gegen) inhibit atherogenic events: A study of the combination effects of the 2-herb formula. Journal of Ethnopharmacology 143(3): 859-866.

7. Kang ting, Chai JD, Xing C, Nan JL, Yang PL, et al. (2010) Danshen protects endothelial progenitor cells from oxidized low-density lipoprotein induced impairment. J Zhejiang Univ-Sci B (Biomed \& Biotechnol) 11(8): 618-626.

8. Gao H, Huang L, Ding F, Yang K, Feng Y, et al. (2018) Simultaneous purification of dihydrotanshinone, tanshinone I, cryptotanshinone, and tanshinone IIA from Salvia miltiorrhiza and their anti-inflammatory activities investigation. Scientific Reports 8(1): 8460-8472.

9. Di Cesare Mannelli L, Piccolo M, Maione F, Ferraro MG, Irace C, et al. (2018) Tanshinones from Salvia miltiorrhiza Bunge revert chemotherapy-induced neuropathic pain and reduce glioblastoma cells malignancy. Biomedicine \& Pharmacotherapy 105: 1042-1049.

10. Tao S, Zheng Y, Lau A, Jaramillo MC, Chau BT, et al. (2013) Tanshinone I Activates the Nrf2-Dependent Antioxidant Response and Protects Against As (III)-Induced Lung Inflammation In Vitro and In Vivo. Antioxidants \& Redox Signaling 19(14): 1647-1661.

11. Zhao J, Lou J, Mou Y, Li P, Wu J, et al.(2011) Diterpenoid Tanshinones and Phenolic Acids from Cultured Hairy Roots of Salvia miltiorrhiza Bunge and Their Antimicrobial Activities. Molecules 16(3): 2259-2267.

12. Yi Gong, Yanli Li,Yin Lu, Linglin Li, Hamid Abdolmaleky, et al. (2011) Bioactive tanshinones in Salvia Miltiorrhiza inhibit the growth of 
prostate cancer cells in vitro and in mice. International Journal of Cancer 129(5): 1045-1052.

13. Wu Y, Ni Z, Shi Q, Dong M, Kiyota H, et al. (2012) Constituents from Salvia Species and Their Biological Activities. Chemical Reviews 112(11): 5967-6026.

14. Hartmann T (2007) From waste products to ecochemicals: fifty years research of plant secondary metabolism. Phytochemistry 68(22-24): 2831-2846.

15. CHEN Shi lin, SUO Feng mei, HAN Jian ping, XIE Cai xiang, YAO Hui, et al. (2007) Analysis on ecological suitability and regionalization of traditional Chinese medicinal materials. Chinese Traditional and Herbal Drugs 38(4): 0481-0487.

16. Chang W, Thissen U, Ehlert KA, Koek MM, Jellema RH, et al. (2006) Effects of Growth Conditions and Processing on Rehmannia glutinosa using Fingerprint Strategy. Planta Medica 72(5): 458-467.

17. Wang Z, Ma P, Xu L, He C, Peng Y, et al. (2013) Evaluation of the content variation of anthraquinone glycosides in rhubarb by UPLC-PDA. Chem Cent J 7(1): 170-176.

18. Ren G, Li L, Hu H, Li Y, Liu C, et al. (2016) Influence of the Environmenta Factors on the Accumulation of the Bioactive Ingredients in Chinese Rhubarb Products. PLOS ONE 11(5): 54-64.

19. Yang S, Li J, Zhao Y, Chen B, Fu C (2011) Harpagoside Variation Is Positively Correlated with Temperature in Scrophularia ningpoensis Hemsl. Journal of Agricultural and Food Chemistry 59(5): 1612-1621.

20. ZHANG Chen lu, LIANG Zong suo, GUO Hong bo, LIU Jing ling, LIU Yan, et al. (2015) Correlation analysis between meteorological factors?biomass?and active components of Salvia miltiorrhiza in different climatic zones. China Journal of Chinese Materia Medica 40(04): 607-613.

21. Yange Yu (2017) Study on the analyses of ecological factors related to quality difference of wild Salvia miltiorrhiza. Zhejiang Sci Tech University 37(02): 289-296.

22. WANG Wei, ZHOU Guo jun, LI Yan, ZHOU Tong shui (2015) Effect of soil moisture on contents of bioactive components in fresh and dried roots of Salvia miltiorrhiza Bage. Anhui Medical and Pharmaceutical Journal 19(02): 248-251.

23. LI Dong, WU Xian Jun, CHEN Xin (2012) Research Of Rosmarinic Acid Biosynthesis Pathway Key Enzyme Gene Expression During Heat Stress In Salvia miltiorrhiza leaves. Journal of Nuclear Agricultural Sciences 26(01): 60-67.

24. YU Yange, ZHANG Xiaodan, HOU Zhuoni, LI Ying, SHEN Chao, et al. (2017) Correlation Analysis of Inorganic Elements in Salvia miltiorrhiza Bunge Growing Wild in China and Its Soil from Different Places. Journal of Zhejiang Sci-Tech University (Natural Sciences) 37(02): 289-296.

25. CHEN Xiao yu, HE Chao, YAN Bin bin, LI Wen bin, GENG Ye ye, et al. (2019) Investigation and analysis on difference of cultivation technique situation of Salvia miltiorrhiza. China Journal of Chinese Materia Medica 44(07): 1314-1320.

26. GAO Shu rui, ZHAO Zhi gang, HOU Jun ling, WANG Wen quan, ZHU Jun Jie, et al. (2014) Optimization of Rapid Ultrasonic Extraction Process of Four Active Components from Salviae Miltiorrhizae Radix et Rhizoma by Uniform Design. Chinese Journal of Experimental Traditional Medical Formulae 20(10): 8-12.

27. Bao Shitan (2000) Chemical Analysis Method for Soil. China Agricultural Scientech Press: Beijing, China, p. 35-56.

28. Di P, Zhang L, Chen J, Tan H, Xiao Y, et al. (2013) 13C Tracer Reveals Phenolic Acids Biosynthesis in Hairy Root Cultures of Salvia miltiorrhiza. ACS Chemical Biology 8(7): 1537-1548.

29. Xu Z, Luo H, Ji A, Zhang X, Song J, et al. (2016) Global Identification of the Full-Length Transcripts and Alternative Splicing Related to Phenolic Acid Biosynthetic Genes in Salvia miltiorrhiza. Frontiers in Plant Science 7: 100 .
30. Yu H, Guo W, Yang D, Hou Z, Liang Z (2018) Transcriptional Profiles of SmWRKY Family Genes and Their Putative Roles in the Biosynthesis of Tanshinone and Phenolic Acids in Salvia miltiorrhiza. International Journal of Molecular Sciences 19(6): 1593.

31. Ma Y, Yuan L, Wu B, Li X E, Chen S, et al. (2012) Genome-wide identification and characterization of novel genes involved in terpenoid biosynthesis in Salvia miltiorrhiza. Journal of Experimental Botany 63(7): 2809-2823.

32. Xing B, Yang D, Yu H, Zhang B, Yan K, et al. (2018) Overexpression of SmbHLH10 enhances tanshinones biosynthesis in Salvia miltiorrhiza hairy roots. Plant Science 276: 229-238.

33. DONG Cai Xia, ZHAO Shi Jie, TIAN Ji Chun, MENG Qing Wei, ZOU Qi (2002) Effects of Different Concentration of $\mathrm{NO}_{3}^{-}$on the Chlorophyll Fluorescence Parameters in Seedling Leaves of High Protein Wheat Cultivars. Acta Agronomica Sin Ica 01: 59-64.

34. De la Rosa TM, Julkunen Tiitto R, Lehto T, Aphalo PJ (2001) Secondary metabolites and nutrient concentrations in silver birch seedlings under five levels of daily UV-B exposure and two relative nutrient addition rates. New Phytologist 150: 121-131.

35. He CE, Lu LL, Jin Y, Wei JH, Christie P (2013) Effects of Nitrogen on Root Development and Contents of Bioactive Compounds in Bunge. Crop Science 53(5): 2028.

36. Lilan Lu, Chune He, Yue Jin, Xingling Zhang, Jianhe Wei (2013) Effects of the applications of phosphorus and potassium fertilizers at different growth stages on the root growth and bioactive compounds of Salvia miltiorrhiza Bunge. Australian Journal of Crop Science 7(10): 15331543.

37. WANG Bin, GUO Ya qin, Tan Xian he, WANG Kang Cai, LIU De Hui, et al. (2009) Effect of Copper and Zinc on the Quality and Security of Salviae Miltiorrhizae Bunge. Chinese Journal of Soil Science 40(1): 171-175.

38. WANG Bin, LIU De-hui, TAN Xian-he, WANG Kang-cai, GUO Ya-qin, et al. (2008) Mechanism of copper and zinc on tanshinones of cultivated Salviae miltiorrhizae. China Journal of Chinese Materia Medica 33(17): 2082-2087.

39. SUN Yu xin, LI Yong ming, LIU De hui (2011) Effects of Mn on Growth and Tanshinones Accumulation of Cultivated Salviae Miltiorrhizae Bunge. Soil 43(01): 95-100.

40. Liu J L, Qi Z H, Hao W F, Zhang CL, Liu F H, et al. (2015) The effects of drought and UV-B radiation on the growth and the phenolic compounds of the Salvia miltiorrhiza Bunge leaf. Acta Ecologica Sinica 35(14): 4642-4650.

41. LANG Zong suo, YANG Dong fang, YANG Zong qi, HAN Rui lian, LIU Xiao lei (2013) Effect of Different Soil Moistures on Tolal Phenolic Acid Composition Accumulation and Relevant Enzymatic Activity of Salvia Miltiorrhiza Lamina. Journal of Zhejiang Sci-tech University 30(4): 573578 .

42. Ghimire BK, Son N, Kim S, Yu CY, Chung I (2017) Evaluating water deficit and glyphosate treatment on the accumulation of phenolic compounds and photosynthesis rate in transgenic Codonopsis lanceolata (Siebold \& Zucc.) Trautv. over-expressing $\gamma$-tocopherol methyltransferase $(\gamma$-tmt) gene. 3 Biotech 7(3): 167-174.

43. Sarker U, Oba S (2018) Drought stress enhances nutritional and bioactive compounds, phenolic acids and antioxidant capacity of Amaranthus leafy vegetable. BMC Plant Biol 18(1): 258-263.

44. Rodriguez Calzada T, Qian M, Strid A, Neugart S, Schreiner M, et al. (2019) Effect of UV-B radiation on morphology, phenolic compound production, gene expression, and subsequent drought stress responses in chili pepper (Capsicum annuum L.). Plant Physiol Biochem 134: 94102.

45. Yang S, Li J, Zhao Y, Chen B, Fu C (2011) Harpagoside Variation Is Positively Correlated with Temperature in Scrophularia ningpoensis Hemsl. Journal of Agricultural and Food Chemistry 59(5): 1612-1621. 
46. GUO Jie, ZHANG Qin, SUN Cheng Zhong, WEN Jian, XIE Cai Xiang (2017) Spatial variations of ginsenosides in Panax ginseng and their impact factors. Chinese Journal of Plant Ecology 41(09): 995-1002.

47. Løvdal T, Olsen KM, Slimestad R, Verheul M, Lillo C (2010) Synergetic effects of nitrogen depletion, temperature, and light on the content of phenolic compounds and gene expression in leaves of tomato. Phytochemistry 71(5-6): 605-613.

48. Martz F, Peltola R, Fontanay S, Duval RE, Julkunen Tiitto R, et al. (2009) Effect of latitude and altitude on the terpenoid and soluble phenolic

\section{ISSN: 2574-1241}

DOI: $10.26717 /$ BJSTR.2020.24.004051

Wenquan Wang. Biomed J Sci \& Tech Res

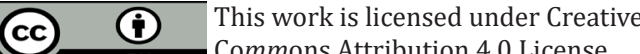

Submission Link: https://biomedres.us/submit-manuscript.php composition of juniper (Juniperus communis) needles and evaluation of their antibacterial activity in the boreal zone. J Agric Food Chem 57(20): 9575-9584.

49. Akerstrom A, Jaakola L, Bang U, Jaderlund A (2010) Effects of latituderelated factors and geographical origin on anthocyanidin concentrations in fruits of Vaccinium myrtillus L. (bilberries). J Agric Food Chem 58(22): 11939-11945 